\title{
Reducing treatment costs for thyroid cancer patients in Japan
}

Japan's healthcare system disincentivises longer hospital stays to reduce costs, but also categorises certain complications as secondary diseases, which incur separate addilonal payments. The high complications makes these costs unavoidable. Hiroki Konno, Associate Professor at the College of Sports Sciences at Nihon University, Japan, studied the implications of this for four prognostic factors affecting thyroid cancer

simple change in approach th will reduce costs for hospitals and for patients.

Tumour has grown into a nearby structure

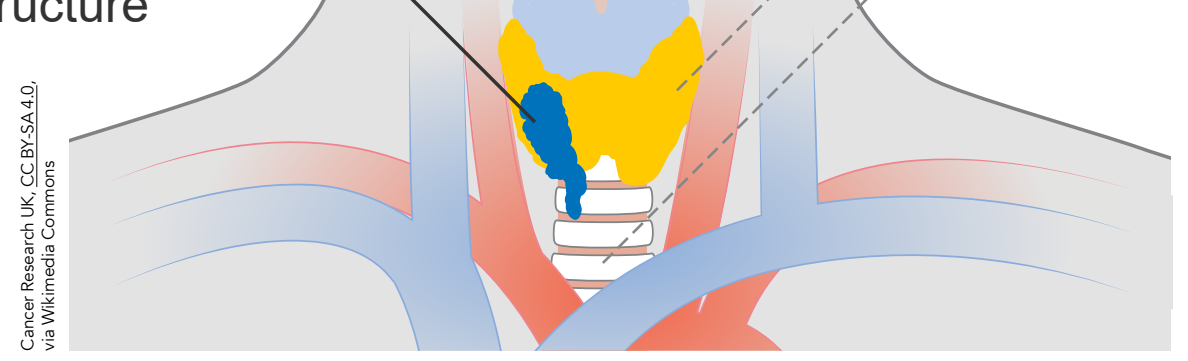

\section{JAPAN'S HEALTHCARE SYSTEM} DPC/PDPS was initially rolled out at 82 in Jpitan. It was estim of all hospitals in Japan. It was estimated that, by ind hospitals and cover more han two-thinds used solely for curative care and not other functions such as rehabilitation, long-term, or palliative care.

DPC/PDPS works by classifying each inpatient event and pre-determining the rate of medical costs, first by their diagnosis and then by the procedures needed to treat their condition. The cost to the patient is then calculated and charged 'per diem', ie, for each day they spend in hospital receiving treatment, rather than per episode. Basic hospitalisation stays examinations, diagnostic imaging, medications, injections, and treatmen under 10,000 yen are included in a categories. Surgery radiagion the anaesthesia, and more complex medical treatments costing more th known as fee-for-service (FFS).

Under the DPC/PDPS, there are incentives for hospitals to minimise the length of time a patient stays ('length of stay' or LOS), as the per diem payment gradually decreases as LOS increases. categories under the system: a higher daily reimbursement charge applies to the early stages of hospitalisation average hospitalisation, and lower daily exceed average LOS. This reducing rate 10,000 yen incur separate charges LOS is divided into three payment averate

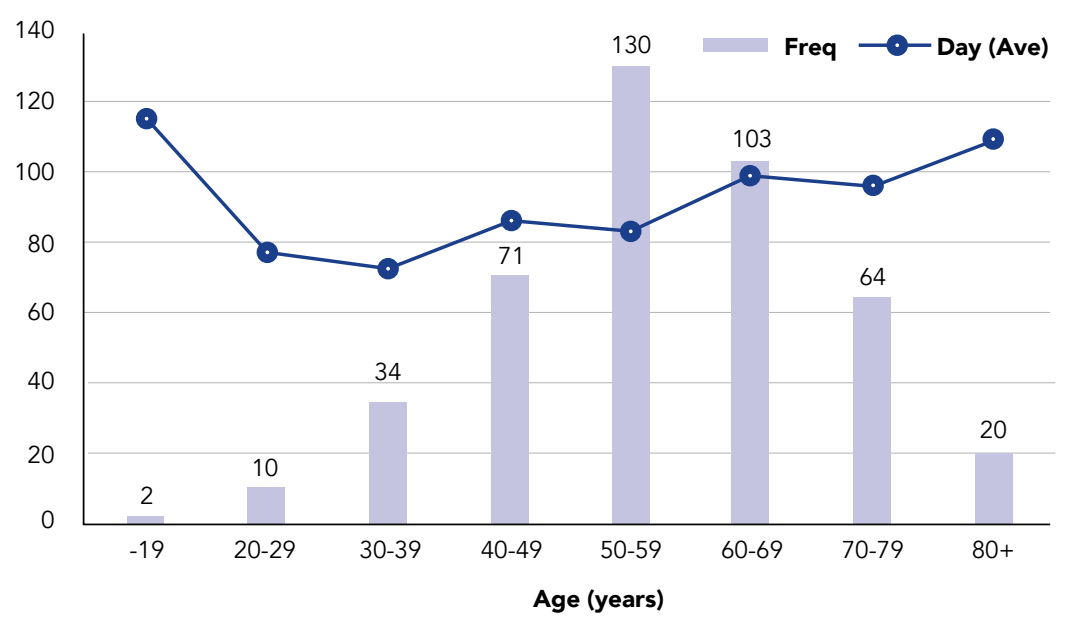

Figure 1. Length of stay in hospital stratified by age.

was introduced after previous studies found it was effective at reducing acknowledgement that reducing LOS should not interfere with the quality of health care patents receive). and patients alike.

\section{PROGNOSTIC FACTORS IN}

THYROID CANCER

Japan has seen a steady decrease in

the mortality rates from thyroid cancer.

found to influence prognosis following a thyroidectomy, including metastasis, lymph nodes, age, hypocalcaemia (low levels of calcium) and hypothyroidism (when the thyroid gland does not produce enough hormones).

Cervical lymph node metastasis is common in patients with thyroid cancer, and is especially high, at $30-80 \%$, in papillary thyroid cancer (cancer arising from follicular cells, which produce and store thyroid hormones). It has previously been reported that metastasis increases the risk of postoperative cancer recurrence. Increasing age is also an important prognostic factor in thyroid cancer, patients younger than 45 years are seen to have tage of their umour, while com the and complication risks also generally increase with age. Hypocalcaemia

is a common post-operative

In April 2014 secondary diseases and
comorbidities were added into the final amount to pay as FFS.

This study aims to provide optima care strategies for thyroid cancer Unavoidable side effects and complications

arising from a patients undergoing thyroidectomy by thyroidectomy and is caused by secondary hypoparathyroidism (decreased secretion of the parathyroid identifying complications prolonging of the parathyroid glands; the parathyroid

reatment are also usually classified as secondary diseases, which means if these conditions arise dun Course of their treatme during the the Japanese Ministry of Health, Labour, and Welfare (MHIW) has also stated that patients with secondary diseases and comorbidities are predicted to have an extended LOS in hospitals, based on data provided by hospitals using DPC/PDPS. This presents something of a catch-22, with the system disincentivising longer hospital stays, while categorising some of the conditions that produce longer stays under FFS; costs for both patients and hospitals increase.

As an expert in the economics of healthcare, Hiroki Konno from Nihon University, Japan, studied the impact cancer to see if new approaches might hormone regulates

\section{hospital stay.}

This is largely due to improvements in treatment, including a combination grands) act radionoval of thyroid glands) and radioactive iodine-131

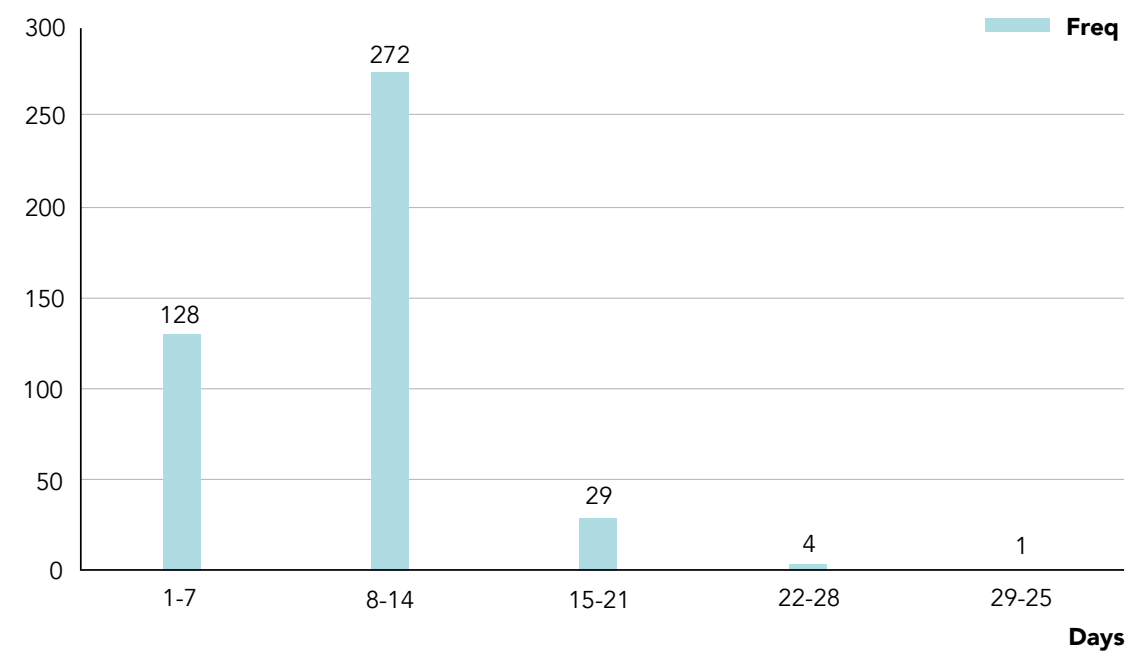



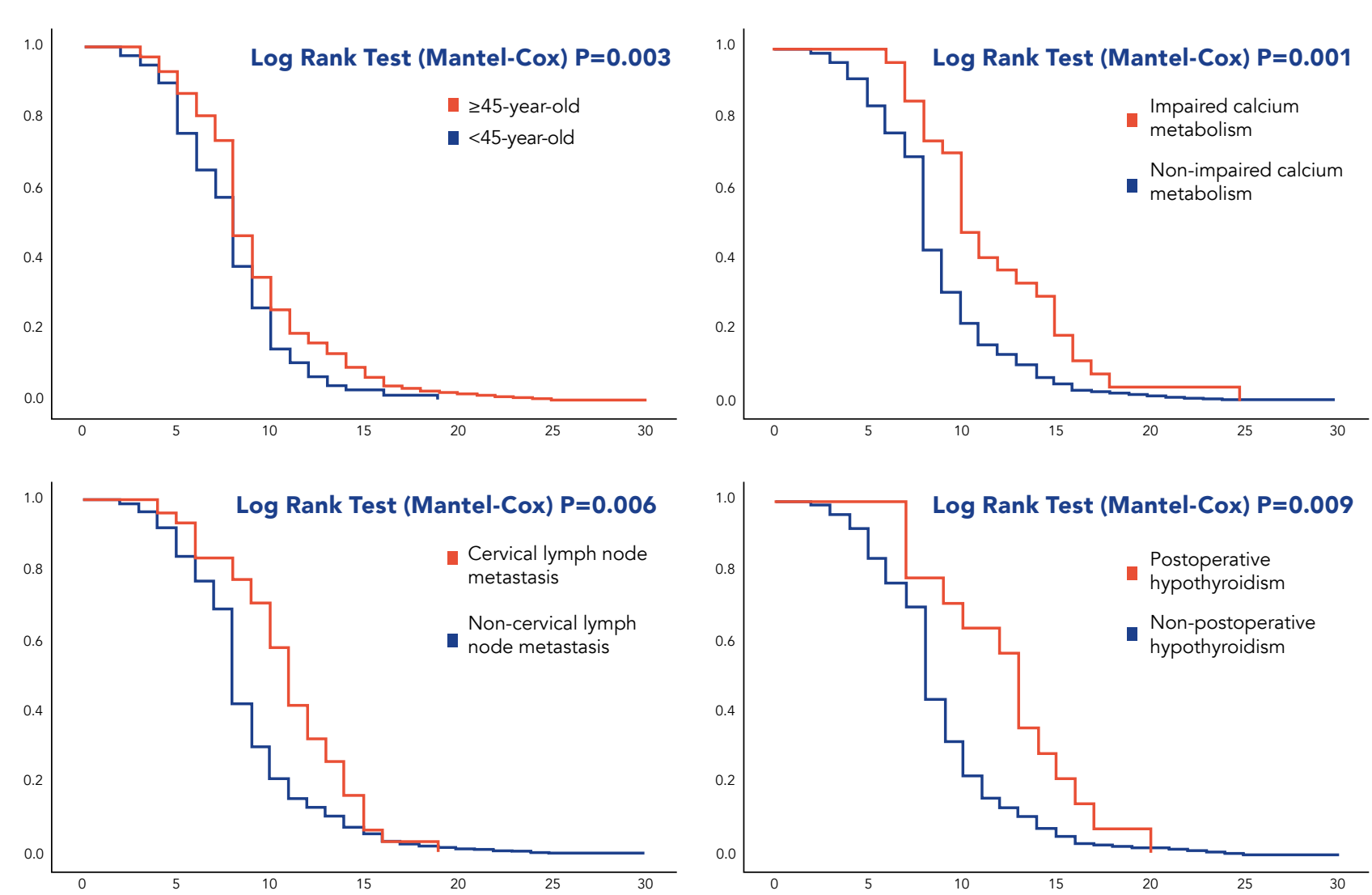

Figure 3. Cumulative probability for fou
and (4) postoperative hypothyroidism.

complications of a thyroidectomy will These prognostic factors have a high risk of occurrence and is out of the hospitals' likely lead to longer LOS in hospitals and inevitably higher costs, both to the patients themselves and in terms of the greater resources needed to treat them

Konno looked specifically at those four key prognostic factors in 434 patients, using their medical and claim records costoperative complications in thyroid they should be classified as prognas 2008. His goal was to determ strategies for

future thyroid

cancer patients

undergoing

Konno's prognostic

longer length of stay in hospitals.

quickly to save resources, Konno's study showed that these four prognostic

factors do indeed cause longer LOS in hospitals. As a result of this study, Konno proposed a solution to this seemingly intractable problem. He believes the key is to identify patients who are likely to develop disorders of calcium metabolism and postopordive hypothyroidism, and

a more appropriate more appropriate hospital bed type,
such as outpatient such as outpatien
care. This would save acute-care costs and means patients could receive better factors under the DPC/PDPS framework and more efficient treatment with the esource and cost implications of postoperative complications. in include numbness, systemic spasms, hallucinations, and severe cases can

Konno found that patients with one of the following prognostic factors were more likely to have a longer hospital stay in an acute-care bed after surgery: Age 45 or older: $14.5 \%$ - Positive for cervical lymph node

Impaired calcium metabolism: $28.79 \%$ Postoperative hypothyroidism: cause heart failure or affect the function of the brain. Hypothyroidism can cause drowsiness, impaired memory, depression, lethargy, local swelling, and hair loss. This long list of symptoms can result in patients staying much longer in

While DPC/PDPS provides an incentive for hospitals to discharge patients more and more efficient treatment with the appropriate resources. Crucially for the incur unnecessary higher charges.

Of course, the importance of this work extends beyond thyroid cancer care. Identifying unavoidable complications from other treatments will enable Japan widely. A revison to DPC/PDPS widely. A revision to DPC/PDPS can providu, LOS, unnecessary costs, and healthcare providers to reconsider

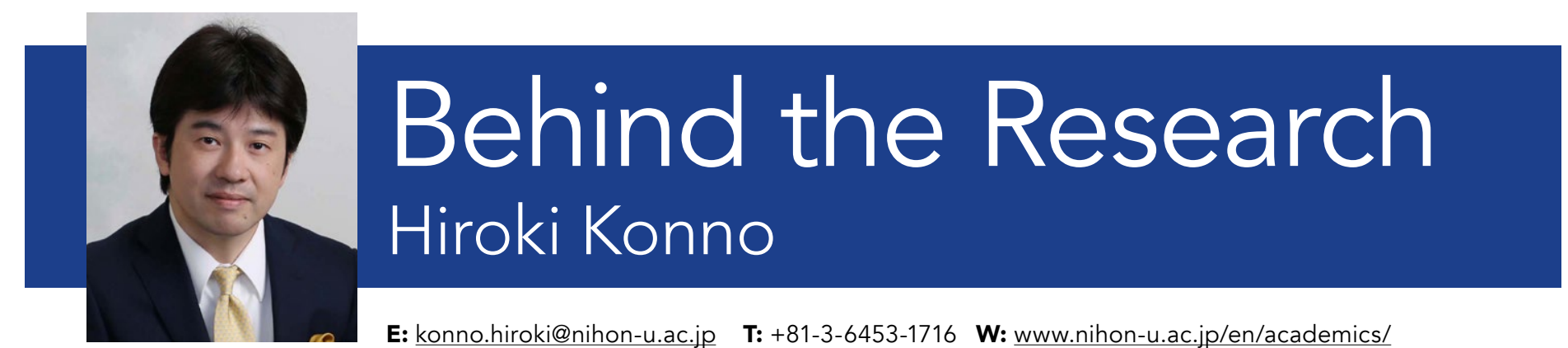

E: konno.hiroki@nihon-u.ac.jp

\section{Research Objectives}

Hiroki Konno specialises in the economics of healthcar cluding medical statistics and the management of

\section{Detail}

\section{Address}

College of Spots

3-34-1 Shimoum

Setagaya-ku

Tokyo 154-8513, Japan

Bio

After a Masters in Economics from Hitotsubashi University, Hiroki worked at the International University of Health and Welfare before becoming associate professor at Nihon University's College of Sports Sciences, where he studies health economics. He won the Encouraging Prize on Health Economics 2005 and has recently published a paper on the importance of promoting medical functional differentiation in Japan, "The importance of Medical Functional Differentiation and Regional Packaged Care $\sim$ after the opportunity of
COVID-19 epidemic".

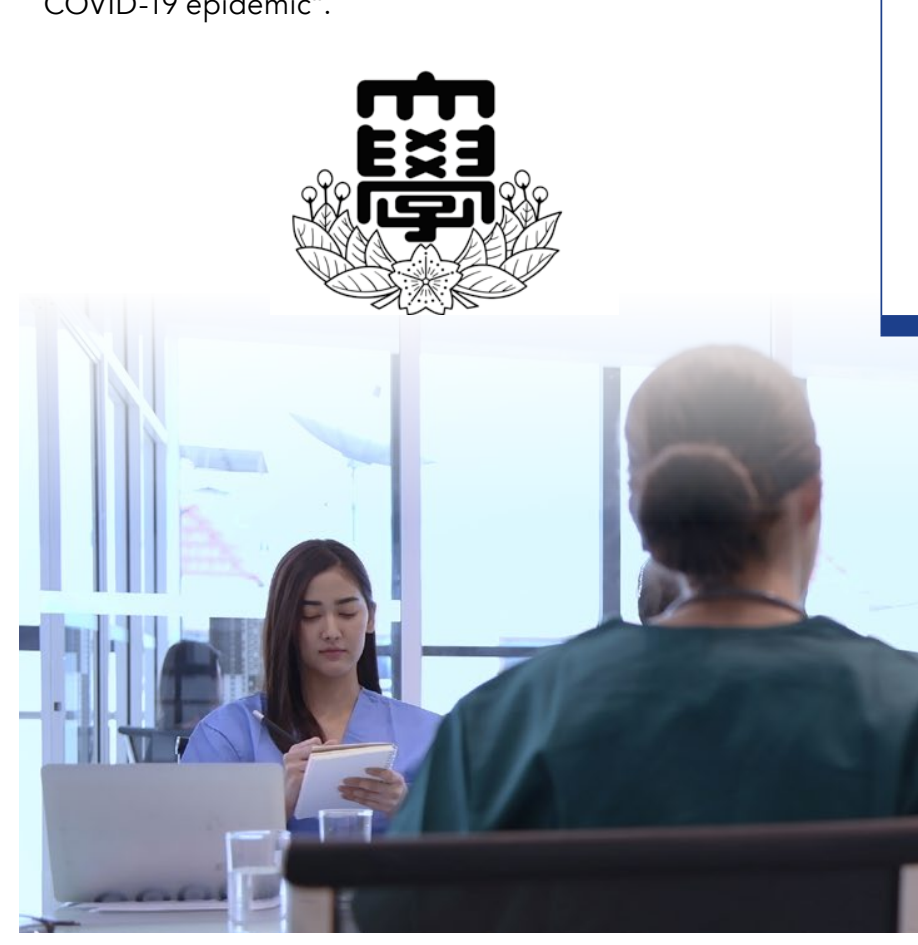

\section{References}

Hamada, H Sekimoto, M \& Imanaka, Y (2012). Effects of the per diem prospective payment system with DRG-like healthcare quality in Japan. Health Policy, 107 (2-3), 194-201. Konno, $\mathrm{H}$ (2021). Prognostic factors in thyroid cancer patients following surgical resection. a case series analysis Presented at the 15th Japan Society for Health Care Management Conference in TOCHIGI 2015.

Zhang, TT Qi, XZ Chen, JP Shi, RL et al (2019). The association between tumor's location and cervical lymph Surgery, 8 (5), 557-568.

\section{Personal Response}

How would you revise DPC/PDPS to avoid the tensions between cost efficiency and appropriat tensions betwe
patient care?

II Highly severe complications that occur after surgery less severe complications are excluded from the DPC/ PDPS regulations because they can be treated by sub-acute care. Since DPC/PDPS is a system for acute care, treatments that can be handled by sub-acute care to secure a limited number of beds for acute care will contribute to cost effectiveness and improved quality of
medical care.

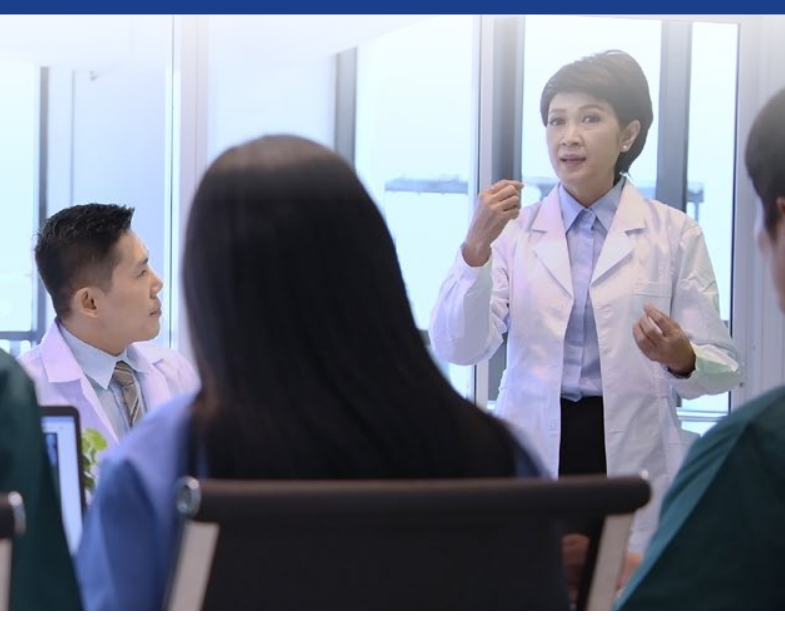

